\title{
RANK ONE PERTURBATION AND ITS APPLICATION TO THE LAPLACIAN SPECTRUM OF A GRAPH
}

\author{
WASIN SO*
}

\begin{abstract}
In this note we characterize those rank one perturbation of symmetric matrices which change only one eigenvalue. Then we apply the result to study how the Laplacian spectrum of a graph change when adding an edge.
\end{abstract}

1. Rank one perturbation. In this section, we study rank one perturbation of symmetric matrices. Given that $A$ and $B$ are symmetric matrices. Let $\alpha_{1} \geq \ldots \geq$ $\alpha_{n}, \beta_{1} \geq \ldots \geq \beta_{n}$ and $\gamma_{1} \geq \ldots \geq \gamma_{n}$ be the spectra of $A, B$ and $A+B$ respectively. Then the Weyl's inequalities are

$$
\gamma_{i+j-1} \leq \alpha_{i}+\beta_{j} \leq \gamma_{i+j-n}
$$

for $1 \leq i, j, i+j-1, i+j-n \leq n$. For proof, we refer to [5]. As a consequence of it, we have the following interlacing theorem.

THEOREM 1.1. If $B$ is a positive semidefinite symmetric matrix of rank one then

$$
\gamma_{1} \geq \alpha_{1} \geq \gamma_{2} \geq \ldots \geq \alpha_{n-1} \geq \gamma_{n} \geq \alpha_{n}
$$

Next we want to characterize those $B$ of rank one such that the spectra of $A$ and $A+B$ overlap in $n-1$ places. That means exactly one eigenvalue of $A$ is changed by the perturbation $B$. Let $A$ be partitioned as

$$
A=\left(\begin{array}{cc}
a & x^{T} \\
x & Z
\end{array}\right)
$$

where $x$ is an $(n-1)$ vector, $x^{T}$ its transpose, and $Z$ is an $(n-1) \times(n-1)$ symmetric matrix.

LEMMA 1.2. If the spectrum of $Z$ is same as $n-1$ eigenvalues of $A$ then $x=0$.

Proof. Suppose the spectrum of $Z$ is $\alpha_{1} \geq \ldots \geq \alpha_{k-1} \geq \alpha_{k+1} \geq \ldots \geq \alpha_{n}$. Then

$$
\operatorname{tr} A^{T} A-\operatorname{tr} Z^{T} Z=\alpha_{k}^{2} \text { and } \operatorname{tr} A-\operatorname{tr} Z=\alpha_{k} .
$$

But, from the partition of $A$, we have

$$
\operatorname{tr} A^{T} A=a^{2}+2 x^{T} x+\operatorname{tr} Z^{T} Z \text { and } \operatorname{tr} A-\operatorname{tr} Z=a .
$$

Consequently, $a=\alpha_{k}$, and so $x^{T} x=0$. This implies $x=0$.

LEMMA 1.3. If the matrix $C=\left(\begin{array}{cc}a+\beta & x^{T} \\ x & Z\end{array}\right)$ with $\beta \neq 0$ has eigenvalues $\left\{\alpha_{1}, \ldots, \alpha_{k}+\beta, \ldots, \alpha_{n}\right\}$ for some $k$ then $x=0$.

\footnotetext{
* Institute for Mathematics and its Applications, University of Minnesota, Minneapolis, MN 55455. na.so@na-net.ornl.gov
} 
Proof. The characteristic polynomials of $A$ and $C$ are

$$
\operatorname{det}(\lambda I-A)=\left(\lambda-\alpha_{1}\right) \cdots\left(\lambda-\alpha_{n}\right)
$$

and

$$
\operatorname{det}(\lambda I-C)=\left(\lambda-\alpha_{1}\right) \cdots\left(\lambda-\alpha_{k}-\beta\right) \cdots\left(\lambda-\alpha_{n}\right)
$$

respectively. Hence we have

$$
\operatorname{det}(\lambda I-C)=\operatorname{det}(\lambda I-A)-\beta\left(\lambda-\alpha_{1}\right) \cdots\left(\lambda-\alpha_{k-1}\right)\left(\lambda-\alpha_{k+1}\right) \cdots\left(\lambda-\alpha_{n}\right) .
$$

¿From the partition of $C$, it follows that

$$
\operatorname{det}(\lambda I-C)=\operatorname{det}(\lambda I-A)-\beta \operatorname{det}(\lambda I-Z)
$$

Since $\beta \neq 0$, we have

$$
\operatorname{det}(\lambda I-Z)=\left(\lambda-\alpha_{1}\right) \cdots\left(\lambda-\alpha_{k-1}\right)\left(\lambda-\alpha_{k+1}\right) \cdots\left(\lambda-\alpha_{n}\right) .
$$

This implies that the spectrum of $Z$ is same as $n-1$ eigenvalues of $A$. It follows that $x=0$ from Lemma 1.2.

THEOREM 1.4. Let $A$ and $B$ be symmetric matrices with spectra (not necessary in descending order) $\left\{\alpha_{1}, \ldots, \alpha_{n}\right\}$ and $\{\beta, 0, \ldots, 0\}$ respectively. If $A+B$ has eigenvalues $\left\{\alpha_{1}+\beta, \alpha_{2}, \ldots, \alpha_{n}\right\}$ then $A B=B A$.

Proof. Without loss of generality, we may assume $B$ to be the diagonal matrix $\operatorname{Diag}(\beta, 0, \ldots, 0)$. If $\beta=0$ then the result is trivially true. For $\beta \neq 0$, from Lemma 1.3, $A$ is of the form $\left(\begin{array}{ll}a & 0 \\ 0 & Z\end{array}\right)$. Hence it is clear that $A B=B A$.

The converse of Theorem 1.4 is obviously true. Actually a more general result is true: Let $H$ and $K$ be (not necessary symmetric) matrices with spectra (not necessary in descending order) $\left\{\alpha_{1}, \ldots, \alpha_{n}\right\}$ and $\left\{\beta_{1}, \ldots, \beta_{n}\right\}$ respectively. If $H K=K H$ then $H+K$ has eigenvalues (relabelling of $\beta$ 's if necessary) $\left\{\alpha_{1}+\beta_{1}, \ldots, \alpha_{n}+\beta_{n}\right\}$. In view of Theorem 1.4, it is tempting to conjecture that the converse (at least for symmetric matrices) of this statement is also true. However we have the following example.

Take $H=\left(\begin{array}{ccc}0 & 0 & 0 \\ 0 & 6-\sqrt{2} & 2 \\ 0 & 2 & 4+2 \sqrt{2}\end{array}\right)$ and $K=\left(\begin{array}{ccc}4 & 0 & 0 \\ 0 & -4 & 0 \\ 0 & 0 & 0\end{array}\right)$. Both are symmetric and have eigenvalues $\{8,2+\sqrt{2}, 0\}$ and $\{4,0,-4\}$ respectively. $H+K$ has eigenvalues $\{6+\sqrt{2}, 4,0\}$, but $H K \neq K H$.

2. The Laplacian spectrum of a graph. Let $G=(V, E)$ be a simple graph on $n$ vertices, where $V$ is the set of vertices and $E$ is the set of edges. The Laplacian matrix of $G$ (with respect to an ordering of $V$ ) is defined as

$$
L(G)=D(G)-A(G)
$$


where $A(G)$ is the adjacency matrix, and $D(G)$ is the degree matrix. We are interested in the spectrum of $L(G)$, which is called the Laplacian spectrum of $G$. Note that the Laplacian spectrum of $G$ is independent of the choice of ordering. There are a number of interesting results and conjectures on the Laplacian spectrum of $G[1,3,6]$. In this section, we want to study the effect on the Laplacian spectrum when adding an edge to $G$.

Let $G^{+}$denote the graph by adding an edge $\{i, j\}$ to $G$. With a suitable ordering, we have

$$
L\left(G^{+}\right)=L(G)+K
$$

where $K=\left(\begin{array}{cc}1 & -1 \\ -1 & 1\end{array}\right) \oplus 0_{n-2}$. Since $K$ is a positive semidefinte symmetric matrix with rank equal to one, by Theorem 1.1, we have the following "Laplacian Folklore".

THEOREM 2.1. The $n-1$ largest eigenvalues of $L(G)$ interlace the eigenvlaues of $L\left(G^{+}\right)$.

Next result gives more information. For $u \in V$, define $\operatorname{Adj}(u)=\{v \in V:\{u, v\} \in$ $E\}$.

THEOREM 2.2. Adj $(i)=\operatorname{Adj}(j)$ if and only if the spectrum of $L\left(G^{+}\right)$overlaps the spectrum of $L(G)$ in $n-1$ places.

Proof. By Theorem 1.4, the spectrum of $L\left(G^{+}\right)$overlaps the spectrum of $L(G)$ in $n-1$ places iff $L(G) K=K L(G)$. Then the result follows from the observation that $\operatorname{Adj}(i)=\operatorname{Adj}(j)$ iff $L(G) K=K L(G)$.

Harary and Schwenk [4] studied those graphs $G$ such that $A(G)$ has integral spectrum. Similar problem for $L(G)$ is also interesting [2]. We call $G$ Laplacian integral if $L(G)$ has an integral spectrum. We list some examples of Laplacian integral graphs.

1. Complete graphs, $K_{n}$ for all $n$.

2. Bipartite graphs, $K_{n, m}$ for all $n, m$.

3. Star graphs, $S_{n}$ for all $n$.

4. Path graphs, $P_{n}$ for $n \leq 3$.

5. Cycle graphs, $C_{n}$ for $n \leq 4$.

6. Wheel graphs, $W_{n}$ for $n \leq 5$.

For the rest of this section, we concentrate on the problem of preserving Laplacian integrality by adding an edge. Note that $K$ is a positive semidefinite symmetric matrix with trace equal to 2. In order to preserve Laplacian integrality, the perturbation $K$ must move up either (a) one eigenvlue of $L(G)$ by 2 or (b) each of two eigenvalues of $L(G)$ by 1 . For case (a), it means that the spectrum of $L\left(G^{+}\right)$overlaps the spectrum of $L(G)$ in $n-1$ places. Hence Theorem 2.2 characterizes this situation and we have the following sufficient condition for preserving Laplacian integrality by adding an edge.

Corollary 2.3. If $A d j(i)=A d j(j)$ then $G$ is Laplacian integral iff $G^{+}$is Laplacian integral.

Easy examples on graph of three vertices show that such sufficient condition is not necessary. It is well expected because of the presence of case (b). Hence it is highly desirable to characterize the situation described in (b). Some progress has been made by Grone and Merris [2]. However, as far as I know, it is still open. 


\section{REFERENCES}

[1] R. GRone, On the geometry and Laplacian of a graph, Linear Algebra and its Applications, 150 (1991), pp. 167-178.

[2] R. Grone And R. MerRis, The Laplacian spectrum of a graph II, Preprint, (1992).

[3] R. Grone, R. Merris, And V. S. Sunder, The Laplacian spectrum of a graph, SIAM Journal on Matrix Analysis and Applications, 11 (1990), pp. 218-238.

[4] F. HaRRAY AND A. SchWEnk, Which graphs have integral spectra, in Graphs and Combinatorics, F. H. R.A. Bari, ed., Springer-Verlag, Berlin, 1974, pp. 45-51.

[5] R. Horn and C. R. Johson, Matrix Analysis, Cambridge, 1985.

[6] R. MERRIs, Laplacian matrices of graphs: A survey, Preprint, (1992). 